\title{
Angle-resolved Valence EELS of a Single Crystal Gold Sample
}

\author{
Marek Malac ${ }^{1,2}$, Koji Kimoto ${ }^{4}$, Ray Egerton ${ }^{1,2}$, Prashant Shekhar ${ }^{3}$, Zubin Jacob $^{3}$, Yoshifumi Taniguchi ${ }^{5}$, \\ Vaibhav Gaind ${ }^{6}$
}

1. National Institute of Nanotechnology, 11421 Saskatchewan Drive, Edmonton, Canada

2. Department of Physics, University of Alberta, T6G 2E1, Edmonton, Canada

3. ECE, University of Alberta, Canada

4. NIMS, 1-1 Namiki, Tsukuba, Ibaraki 305-0044, Japan

5. Hitachi High-Technologies Corp., 882, Ichige, Hitachinaka-shi, Ibaraki-ken, 312-8504, Japan

6. KLA-Tencore, One Technology Drive, Milpitas, California, 95035, USA

Electron energy loss spectroscopy (EELS) in a TEM can measure the optical response of individual nanostructures at high spatial resolution [1] and obtain structure and morphology of the individual nanostructures. Here we show that high quality valence EELS (VEELS) can be acquired and interpreted to well below $2 \mathrm{eV}$ in an instrument equipped with a cold field emission gun (CFEG) and a standard Gatan Image Filter (GIF) TridiemTM. We provide interpretation of the main spectral feature in the momentum-resolved EELS (qEELS) spectrum.

The data was obtained in a Hitachi HF 3300 TEM/ STEM with a GIF 863 TridiemTM in $q$ EELS mode at 300 $\mathrm{keV}$ incident energy. For comparison, data was obtained from the same sample using a monochromated FEI Titan with GIF Quantum ${ }^{\mathrm{TM}}$ ERS operated at $80 \mathrm{keV}$. A standard $12 \mathrm{~nm}$ thick single crystal gold with beam along [001] direction was used. The full width at half maximum (FTHM) and at tenth maximum (FWTM) can be used to measure instrument suitability for VEELS. Using the CFEG instrument we obtained FWHM $\approx 480 \mathrm{meV}$ and FWTM $\approx 1 \mathrm{eV}$. The monochromated instrument delivered FWHM $\approx 85 \mathrm{meV}$ and FWTM $\approx 230 \mathrm{meV}$ indicating very narrow electron energy distribution. The sample was present in the beam.

Fig. 1 shows an angle-resolved ( $\omega-q$ map) VEELS obtained from the CFEG instrument. Fig. 2 shows the corresponding angle-integrated VEEL spectra from both the monochromated and CFEG instruments. Fig. 2 shows that by selecting an angular range very slightly off the forward scattering axis results in clearly observable features down to about $1.5 \mathrm{eV}$ in the CFEG instrument despite fact that the FWHM of the energy distribution is $\sim 480 \mathrm{meV}$ in the CFEG instrument. The ability to improve the visibility of the VEELS features by going off-axis is in agreement with [1]. In the monochromated instrument (thin lines) the angular integration imposed by the large convergence angle $\left(\approx 2.3 \mathrm{~nm}^{-1}\right)$ of the incident beam in a single-Wien filter instrument results in a "washing" of the spectral features in the mono spectra. The angular integration in the monochromated instrument outweighs the advantage of the narrow energy distribution of the monochromated electron source.

In addition to the ability to measure VEELS to below $2 \mathrm{eV}$, the $\omega-q$ map shown in Fig. 1 allows to identify the origin of VEELS excitations from the shape of their dispersion curves. For example, Cerenkov radiation and surface plasmons can be often clearly distinguished in angle-resolved VEELS of semiconductors [2]. Gold is often used to fabricate optically active nanoparticle assemblies and optically active heterostructures [3]. However the understanding of their properties is challenging since only dipole regime optical data is available [4,5]. Simulations in Fig. $3[5,6]$ show the scattering probability for a $\Delta q$ range as in the CFEG data in Fig. 2 together with calculated surface loss function. Fig. 4 shows the optical density of states calculated for a $12 \mathrm{~nm}$ thick Au slab modelled with [7]. The strong feature at $\sim 2.6 \mathrm{eV}$ in Fig. 1 and Fig. 4 can be interpreted by an extended Drude model as a result of interband transitions at $2.6 \mathrm{eV}$. The conventional surface plasmon resonance of gold is shifted from $\operatorname{Re}(\varepsilon)=-1$ due to interband transitions.

We reported that a CFEG instrument used in momentum-resolved EELS mode provides a convenient way to measure valence EELS excitations to below $2 \mathrm{eV}$. Measuring the $\omega$-q map makes it possible to use the dispersion relation to interpret the origin of spectral features. 


\section{References}

[1] M. Stöger-Pollach, Micron 39, p. 1092.

[2] P.A. Midgley, Ultramicroscopy 76 (1999), p. 91.

[3] K.K. Lance et. al., J. Phys. Chem. B 107 (2003), p.668 .

[4] F.J. Garcia de Abajo, Rev. Mod. Phys. 82 (2010), p. 209.

[5] P. B. Johnson and R. W. Christy, Phys. Rev. B 6 (1972), p. 4370.

[6] J.P.R. Bolton and M. Chen, J. of Phys. Cond. Matter. 7 (1995), p. 3373.

[7] P. G. Etchegoin, E. C. Le Ru, and M. Meyer, J. Chem. Phys. 125, 164705 (2006)

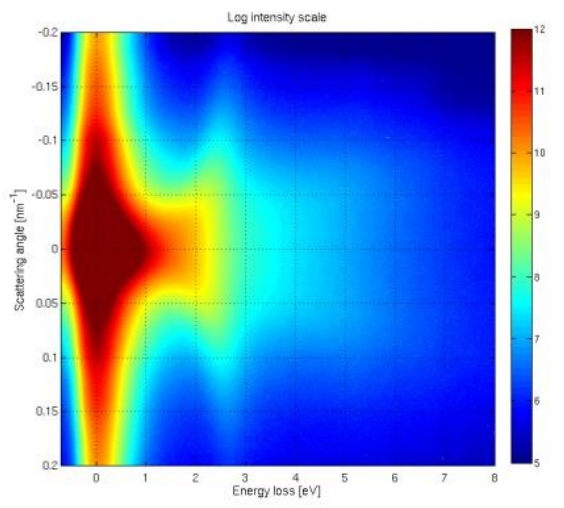

Fig. 1. Angle-resolved VEELS of XX nm thick Au single crystal with angular dispersion $\sim 3.7 \times 10^{-4} \mathrm{~nm}^{-1} / \mathrm{pix}$ and energy step about $10 \mathrm{meV} / \mathrm{ch}$. The raw data is available for download from www.tem-eels.com.

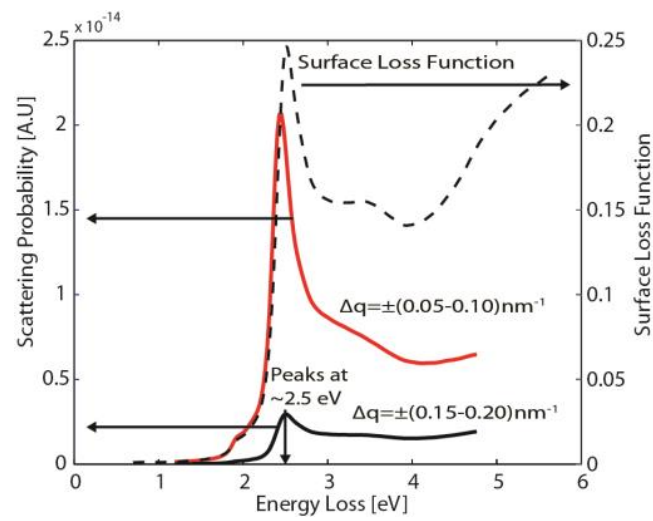

Fig. 3. Scattering probability for $\Delta q$ range shown in Fig. 2 for CFEG instrument (thick lines) using the method of Bolton and Chen [6]. Surface loss function shown in dashed curve.

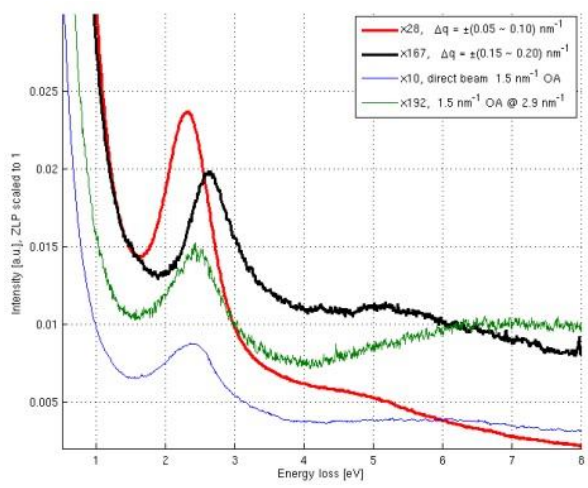

Fig 2. Angle-integrated spectra. Thick lines are for CFEG taken from Fig 1 over $\Delta q$ indicated in the legend. The monochromated instrument spectra (thin lines) were collected with $\Delta q=2.3 \mathrm{~nm}^{-1}$ range centered around the direct beam (blue) and $2.9 \mathrm{~nm}^{-1}$ (green). The zero loss peaks of all spectra were scaled to 1 at $q=0$.

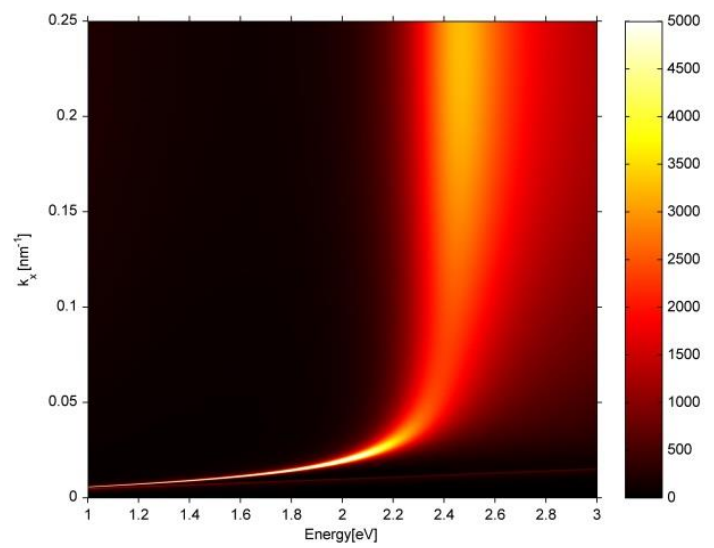

Fig. 4. Photonic density of states for a $12 \mathrm{~nm}$ thick $\mathrm{Au}$ film computed using the model used in [7]. 